\title{
THE SPECTRUM OF THE LAPLACIAN ON A MANIFOLD OF NEGATIVE CURVATURE. I
}

\author{
MARK A. PINSKY
}

\section{Introduction}

Let $M$ be a simply connected complete two-dimensional Riemannian manifold. On $M$ we have the Laplacian, a self-adjoint negative semidefinite operator on the Hilbert space $L^{2}(M)$. In case the curvature is everywhere $\leq-k^{2}<0$, it has been shown [1] that $\lambda_{1} \geq k^{2} / 4$, where $\lambda_{1}$ is the lower bound of the spectrum of the negative Laplacian.

The purpose of this note is to determine more accurate bounds for $\lambda_{1}$. We assume the following conditions on $M$ :

(A) $M$ possesses a global system of geodesic polar coordinates with respect to some point $0 \in M$.

Thus we are considering $R^{2}$ with a Riemannian metric of the form $d s^{2}=$ $d r^{2}+G(r, \theta)^{2} d \theta^{2}$ where $G\left(0^{+}, \theta\right)=0, G_{r}\left(0^{+}, \theta\right)=1$.

(B) $G_{r}(r, \theta) \geq 0 ; G(r, \theta) \geq g(r)$ where $g(r)$ is nondecreasing with $\lim _{r \rightarrow \infty} g(r)$ $=\infty$.

Both of these conditions are satisfied in case the curvature is everywhere nonpositive. Finally we need the technical condition

(C) $\left|\left(G_{r r} / G_{r}\right)_{\theta}\right| \leq$ const, when $r \geq r_{0}, 0 \leq \theta \leq 2 \pi$.

Our main result states that

$$
\inf _{M}\left(G_{r} / G\right) \leq \sqrt{4 \lambda_{1}} \leq \inf _{0 \leq \theta \leq 2 \pi} \varlimsup_{r \rightarrow \infty}\left(G_{r r} / G_{r}\right) .
$$

This result shows, for instance, that when the curvature is constant and equal to $-k^{2}$, then $\lambda_{1}=\frac{1}{4} k^{2}$; no explicit calculations with special functions are needed in our approach.

To prove the lower half of (1.1) we modify the methods used in [1]. To obtain the upper bound we first obtain a comparison function and apply the variational characterization of $\lambda_{1}$. It is shown that if $G_{r r} / G_{r}$ satisfies an upper bound on a sufficiently thick sector, then a corresponding upper bound can be obtained.

\footnotetext{
Received February 4, 1976, and, in revised form, June 3, 1976. Research supported by the National Science Foundation Grant No. MPS71-02838-A04. The author is indebted to $\mathbf{R}$. Osserman for a critical reading of an earlier version of this paper.
} 


\section{The lower bound}

To prove the lower bound, we recall the variational characterization of $\lambda_{1}$ :

$$
\lambda_{1}=\inf _{f \neq 0} \frac{\int_{0}^{2 \pi} \int_{0}^{\infty}\left(f_{r}^{2}+f_{\theta}^{2} / G^{2}\right) G d r d \theta}{\int_{0}^{2 \pi} \int_{0}^{\infty} f^{2} G d r d \theta},
$$

where the infimum is taken over continuous, piecewise $C^{1}$ functions $f$ with compact support. We assume that $G_{r} / G \geq \delta>0$ (if $\delta=0$ there is nothing to prove). Following [1] we have

$$
\int_{0}^{\infty} f^{2} G d r \leq \frac{1}{\delta} \int_{0}^{\infty} f^{2} G_{r} d r=-\frac{2}{\delta} \int_{0}^{\infty} f f_{r} G d r .
$$

Therefore by Schwarz's inequality,

$$
\left(\int_{0}^{\infty} f^{2} G d r\right)^{2} \leq \frac{4}{\delta^{2}}\left(\int_{0}^{\infty} f^{2} G d r\right)\left(\int_{0}^{\infty} f_{r}^{2} G d r\right),
$$

with the conclusion

$$
\int_{0}^{\infty} f_{r}^{2} G d r \geq \frac{\delta^{2}}{4} \int_{0}^{\infty} f^{2} G d r .
$$

When we add in the angular term, do the angular integration, and divide by the denominator of (2.1), we see that for any $f \neq 0$ this quotient is bounded below by $\delta^{2} / 4$, which was to be proved.

\section{The upper bound}

The main result of the section is

Lemma 3.1. Assume that $G_{r r} / G_{r} \leq m$ for $R_{0} \leq r \leq R_{1}, \alpha \leq \theta \leq \beta$. Then

$$
\lambda_{1} \leq \frac{m^{2}}{4}+\frac{\pi^{2}}{\left(R_{1}-R_{0}\right)^{2}}+\frac{\pi^{2}}{(\beta-\alpha)^{2} g\left(R_{0}\right)^{2}} .
$$

Proof. Let

$$
f(r, \theta)=\exp \left(-\frac{1}{2} m r\right) \sin \frac{\pi\left(r-R_{0}\right)}{R_{1}-R_{0}} \sin \frac{\pi(\theta-\alpha)}{\beta-\alpha}
$$

in the indicated region, and let $f=0$ elsewhere. By direct computation $f$ is a solution of the differential equation

$$
f_{r r}+m f_{r}+\left[m^{2} / 4+\pi^{2} /\left(R_{1}-R_{0}\right)^{2}\right] f=0,
$$

with the end condition $f\left(R_{0}, \theta\right)=0, f\left(R_{1}, \theta\right)=0$. Thus 


$$
f_{r r}+\left(G_{r} / G\right) f_{r}+\left[m^{2} / 4+\pi^{2} /\left(R_{1}-R_{0}\right)^{2}\right] f^{\prime}=\left(G_{r} / G-m\right) f_{r} .
$$

Multiply (3.2) by $f G$ and integrate on $\left(R_{0}, R_{1}\right)$; thus

$$
-\int_{R_{0}}^{R_{1}} f_{r}^{2} G d r+\left[\frac{m^{2}}{4}+\frac{\pi^{2}}{\left(R_{1}-R_{0}\right)^{2}}\right] \int_{R_{0}}^{R_{1}} f^{2} G d r=\int_{R_{0}}^{R_{1}}\left(G_{r}-m G\right) f f_{r} d r .
$$

We now integrate the right-hand member of (3.3) by parts. The boundary term is zero, and the new integrand has the same sign as $m G_{r}-G_{r r}$ which is nonnegative by assumption. Therefore

$$
\int_{R_{0}}^{R_{1}} f_{r}^{2} G d r \leq\left[\frac{m^{2}}{4}+\frac{\pi^{2}}{\left(R_{1}-R_{0}\right)^{2}}\right] \int_{R_{0}}^{R_{1}} f^{2} G d r .
$$

To treat the $\theta$-terms in (2.1) we note that $f$ also satisfies $f_{\theta \theta}+\left(\pi^{2} /(\beta-\alpha)^{2}\right) f=0$ with $f(r, \alpha)=0=f(r, \beta)$. Multiplying this equation by $f$ and integrating on $(0,2 \pi)$ we have

$$
\int_{0}^{2 \pi} f_{\theta}^{2} d \theta=\frac{\pi^{2}}{(\beta-\alpha)^{2}} \int_{0}^{2 \pi} f^{2} d \theta .
$$

By hypothesis (B) we can make the following estimations:

$$
\begin{aligned}
\int_{R_{0}}^{R_{1}} \int_{\alpha}^{\beta}\left(f_{\theta}^{2} / G\right) d \theta d r & \leq g\left(R_{0}\right)^{-1} \int_{R_{0}}^{R_{1}} \int_{\alpha}^{\beta} f_{\theta}^{2} d \theta d r \\
& =\pi^{2}(\beta-\alpha)^{-2} g\left(R_{0}\right)^{-1} \int_{R_{0}}^{R_{1}} \int_{\alpha}^{\beta} f^{2} d \theta d r \\
& \leq \pi^{2}(\beta-\alpha)^{-2} g\left(R_{0}\right)^{-2} \int_{R_{0}}^{R_{1}} \int_{\alpha}^{\beta} f^{2} G d \theta d r .
\end{aligned}
$$

Combining this with (3.4) gives

$$
\begin{aligned}
& \int_{\alpha}^{\beta} \int_{R_{0}}^{R_{1}}\left(f_{r}^{2}+f_{\theta}^{2} / G^{2}\right) G d r d \theta \\
& \quad \leq\left[\frac{m^{2}}{4}+\frac{\pi^{2}}{\left(R_{1}-R_{0}\right)^{2}}+\frac{\pi^{2}}{(\beta-\alpha)^{2} g\left(R_{0}\right)^{2}}\right] \int_{\alpha}^{\beta} \int_{R_{0}}^{R_{1}} f^{2} G d r d \theta .
\end{aligned}
$$

Inserting the above $f$ into the variational characterization (2.1), we have proved the lemma.

We can now turn to the proof of the upper half of (1.1). For this purpose, let $\bar{m}=\inf _{\theta} \varlimsup_{r \rightarrow \infty}\left(G_{r r} / G_{r}\right)$. Given $\varepsilon>0$ we can find $R_{0}^{\prime}>0$ and $(\alpha, \beta)$ such that $G_{r r} / G_{r} \leq \bar{m}+\varepsilon$ when $r \geq R_{0}^{\prime}$ and $\alpha \leq \theta \leq \beta$. Let $R_{0}$ be such that $g\left(R_{0}\right)>$ $\pi /[\varepsilon(\beta-\alpha)]$ and $R_{0} \geq R_{0}^{\prime}$. Therefore for any $R_{1}>R_{0}$ we have, by Lemma 3.1,

$$
\lambda_{1} \leq \frac{1}{4}(\bar{m}+\varepsilon)^{2}+\pi^{2} /\left(R_{1}-R_{0}\right)^{2}+\varepsilon^{2} .
$$


In this inequality we let $R_{1} \rightarrow \infty$. Since the resulting inequality is valid for every $\varepsilon>0$, we have proved that $\lambda_{1} \leq \frac{1}{4} \bar{m}^{2}$.

\section{Discussion of the result-applications and examples}

In previous works upper and lower bounds for $\lambda_{1}$ were obtained in terms of the curvature. Recall that in a system of geodesic polar coordinates we have

$$
-K=G_{r r} / G
$$

where $K$ is the Gaussian curvature. Thus we obtain the two-dimensional case of [1]:

Corollary 4.1. Suppose that $K \leq-k^{2}<0$ on $M$. Then $\lambda_{1} \geq \frac{1}{4} k^{2}$.

Proof. $G$ satisfies the inequality $G_{r r} \geq k^{2} G$ with $G\left(0^{+}, \theta\right)=0, G_{r}\left(0^{+}, \theta\right)=$ 1. Thus $h=G_{r} / G$ satisfies the inequality $h_{r}+h^{2} \geq k^{2}$, $\lim _{r \rightarrow 0} r h(r, \theta)=1$. Therefore $h(r, \theta) \geq k$ coth $k r$ with the conclusion that $\left(G_{r} / G\right) \geq k$ everywhere. Applying the lower bound of (1.1), we obtain the stated result.

In [2] it was proved that $K \geq-k^{2}$ implies $\lambda_{1} \leq \frac{1}{4} k^{2}$. Although our method does not yield this result, we do obtain a related localized result.

Corollary 4.2. Suppose that $k^{2}(\theta)=\lim _{r \rightarrow \infty}(-K(r, \theta))$ exists for $\theta \in(\alpha, \beta)$ and satisfies $k^{2}(\theta) \leq k^{2}$. Then $\lambda_{1} \leq \frac{1}{4} k^{2}$.

Proof. We have $G_{r r} / G_{r}=(-K) / h$ where $h=G_{r} / G$ is a solution of the equation $h_{r}+h^{2}=-K$. Using a comparison estimate when $r \rightarrow \infty$ [3] we have $\lim _{r \rightarrow \infty} h(r, \theta)=k(\theta)$. (This also follows by an appropriate use of the Rauch comparison theorem.) Therefore $\lim _{r \rightarrow \infty}\left(G_{r r} / G_{r}\right)=k(\theta)$. Applying the upper half of (1.1), we see that $\lambda_{1} \leq \frac{1}{4} k^{2}$, which was to be proved.

Using the same idea, we can obtain another variation of Cheng's result.

Corollary 4.3. Suppose that $K(r, \theta) \geq-k^{2}$ and $\left(G_{r} / G\right)(r, \theta) \geq k_{1}$ for $\theta \in(\alpha, \beta)$ and $r \geq R_{1}$. Then $\lambda_{1} \leq \frac{1}{4} k^{2} / k_{1}$.

Proof. In this case we have $G_{r r} / G_{r} \leq k^{2} / k_{1}$ for $\theta \in(\alpha, \beta)$ and $r \geq R_{1}$, whence the result follows.

From (1.1) we see that the upper bound depends only on the details of the metric in a neighborhood of infinity. It is natural to ask whether a lower bound can be obtained which only depends on the metric in a neighborhood of infinity. The following example shows, in particular, that in the lower bound part of (1.1), the infimum cannot be replaced by lim inf when $r \rightarrow \infty$.

Example 4.4 Let $K(r)$ be a $C^{\infty}$ function such that

$$
\begin{array}{ll}
K(r)=-1, & 0 \leq r \leq R_{1}, \\
K(r)=-4, & R_{1}+1 \leq r<\infty
\end{array}
$$

where $R_{1}>\pi \sqrt{4 / 3}$; let $G(r)$ be the solution of $G_{r r}+K G=0, G\left(0^{+}\right)=0$, 
$G_{r}\left(O^{+}\right)=1$. Thus $G(r)=\sinh r$ and $G_{r r} / G_{r}=\tanh r$ for $0 \leq r \leq R_{1}$. Following the proof of Lemma 3.1 we let $f(r)=\exp \left(-\frac{1}{2} m r\right) \sin \left(\pi r / R_{1}\right)$ for $0 \leq r \leq R_{1}$ and $f=0$ for $r>R_{1}$, where $m=\tanh R_{1}$. Substituting in the variational characterization (1.1), we have

$$
\lambda_{1} \leq \frac{1}{4}\left(\tanh R_{1}\right)^{2}+\pi^{2} / R_{1}^{2}<1 .
$$

On the other hand, it is clear from (4.2) that $\lim _{r \rightarrow \infty}\left(G_{r} / G\right)$ exists and is equal to 2. Hence $\liminf _{r \rightarrow \infty}\left(G_{r} / G\right)=2>\sqrt{4 \lambda_{1}}$.

By modifying the constants in Example 4.4 and taking $R_{1}$ sufficiently large, we obtain the following proposition: Given $0<a<b$, there exists a rotationally invariant metric $G(r)$ with curvature function $K(r)$ such that $\lim _{r \rightarrow \infty}[-K(r)]=b^{2}$ and $\lambda_{1}<\frac{1}{4} a^{2}$.

It is natural to ask if, under suitable regularity conditions, $\lambda_{1}$ depends only on the details of the metric in a neighborhood of infinity. We have the following result.

Corollary 4.5. Assume that $G_{r} / G$ is nonincreasing along each ray and that $\lim _{r \rightarrow \infty} K(r, \theta)=-k^{2}(\theta)$ exists for each $\theta \in[0,2 \pi]$. Then $\sqrt{4 \lambda_{1}}=\inf _{0 \leq \theta \leq 2 \pi} k(\theta)$.

Proof. From the proof of Corollary 4.2, we have $\lim _{r \rightarrow \infty}\left(G_{r r} / G_{r}\right)=k(\theta)$. On the other hand, the same argument shows that $\lim _{r \rightarrow \infty}\left(G_{r} / G\right)=k(\theta)=\inf _{r>0}\left(G_{r} / G\right)$. Hence the left- and right-hand members of (1.1) are equal in this case, and the proof is complete.

\section{References}

[1] H. P. McKean, An upper bound for the spectrum of $\Delta$ on a manifold of negative curvature, J. Differential Geometry 4 (1970) 359-366.

[2] S. Y. Cheng, Eigenvalue comparison theorems and its geometric applications, Math. Z. 143 (1975) 289-297.

[3] M. Pinsky, An individual ergodic theorem for the diffusion on a manifold of negative curvature, Proc. Confer. Stochastic Differential Equations, Academic Press, New York, 1977, 231-240. 
